Short-term media effects include the alterations of sensitivity toward the real violence from the Past

Sergii Tukaiev ${ }^{2 * \S}$, Yurii Havrylets ${ }^{2 *}, V_{\text {olodymyr Rizun }}^{2 *}$, Jürgen Grimm $^{3 *}$, Andreas Enzminger ${ }^{3 *}$, Igor Zyma $^{1 *}$, Mykola Makarchuk ${ }^{1 *}$

${ }^{1}$ National Taras Shevchenko University of Kyiv, Educational and Scientific Centre "The Institute of Biology and Medicine", 64/13, Volodymyrs'ka St., 01601 Kyiv, Ukraine;

${ }^{2}$ National Taras Shevchenko University of Kyiv, Institute of Journalism, 64/13, Volodymyrs'ka St., 01601 Kyiv, Ukraine;

${ }^{3}$ University of Vienna, Faculty of Social Sciences, Waehringer Straße 29, A-1090, Vienna, Austria

*These authors contributed equally to this work

${ }^{\S}$ Corresponding author

Email addresses:

Sergii Tukaiev: tukaev@univ.kiev.ua

Yurii Havrylets, youri1985@gmail.com

Volodymyr Rizun, v.v.rizun@gmail.com

Jürgen Grimm, juergen.grimm@univie.ac.at

Andreas Enzminger, andreas.enzminger@gmail.com

Igor Zyma, igzyma@gmail.com

Mykola Makarchuk, mykola.makarchuk@gmail.com

Abstract. The purpose of this study was to identify the impact of TV news on the perception and processing of the emotional footage of a historical documentary. We noted that the emotional frames taken from TV news stories are relatively weak emotional stimuli compared to the emotional frames of historical documentary. The subjects exposed to the pre-stimulus evaluated the Holocaust pictures as more unpleasant and more activating than those without it. The ERPs recorded during the presentation of the negative (historical) images had various amplitudes of oscillations due to the preliminary affective impact of TV news footage. The amplitude of P300 in frontal zones was bigger for the group without the preliminary exposure to the emotional TV news frames due to the reduced sensitivity to the content of images. In this case, the historical images demanded less attention and less emotional effort for emotional evaluation of visual information, analysis, retrieval of information from memory and semantic processes, namely search for the meaning of the pictures (occipital P300 and LPP). Short-term media effects include alterations of sensitivity toward the emotional content of visual information.

Keywords: event-related potentials, emotion, violence, Holocaust, TV news

\title{
Introduction.
}

One of the most traumatic events in recent history is the Holocaust because of its absolute inhumanity recognized as a manifestation of mass violence. Social representations of the world history demonstrated the crosscultural consensus and recognized the Holocaust as one of the most important events in world history $(8,9,20)$. Levy and Sznaider (18) analyzed the nature of "good and evil" that symbolizes the Holocaust and showed that memories of the Holocaust contribute to the creation of a common European cultural memory (15). Liu and Hilton (21) recognized the role of the memory of the Holocaust in creating, maintaining and changing new identity. Leggewie \& Lang (17) stressed the importance of the Holocaust when it comes to European memory culture. They construct the whole memory culture with the Holocaust in the center of it all. The studies of the Holocaust concern 
the long-term consequences on the survivors including the adaptative challenges in the second and the third generations, such as the changes in the stress-induced level of cortisol in descendants (29).

Collective memory, retention of historical information seemed to have central importance for the social identity. Although in modern society people of different ages recall events of the past but the generations reflect different interpretations of some events. (26). It manifests itself in relation to the violent past - to the genocides in post-genocidal states $(26,31)$. The attitude to such events and the problem of moral dilemmas become of current importance for the society. However, the differences in the assessment of the Holocaust remembrance cultures by Israelis and Europeans are striking. Gavriely-Nuri \& Lachover (5) analyzed obituaries in Israeli newspapers and found evidence for a "cosmopolitan turn in collective memory construction" (5), which is stimulated by the transnationalization of media communication processes and which supports the acceptance of peace-promoting views. Similar views are expressed by Levy and Sznaider $(18,19)$ and Silbermann \& Stoffers $(30)$. Zuckermann $(32,33)$, examining different Holocaust remembrance cultures in Israel and Germany, found on both sides "ideological" instrumentalization, which inhibits a truly cosmopolitan memory.

Measurements of the real reactions to the traumatic past events present a serious challenge for the researches in social and clinical psychology and neuroscience. However, there is no data examining the psychophysiological impact of the Holocaust, whereas most of the experimental studies display acts of aggression in the fictional movies and on TV in general $(6 ; 10,11 ; 12,14)$. The short-term effects of Mass Media are cumulative $(3,7)$ and lead to profound psychophysiological changes. It is known that watching negatively accented mass media causes increasing insusceptibility to aggression and violence. This implies that watching historical documentaries containing real violence and feeling the anguish of the past events impact the emotional condition of individuals to a different extent.

Using such physiological measurements as electroencephalography (EEG) allow identifying links/relationship between the physiological and cognitive outputs of the participants. Watching historical or up-todate video (documentaries, TV news) or images containing real violence and feeling the anguish of the present or past events impacts the emotional condition of individuals to a different extent. Based on the present cognitivephysiological patterns, we will analyse the responses to the TV-mediated historical events. Focusing on emotionally grave and distressful historical experiences, we will investigate the stress factors of up-to-day negative TV news on the history-based media communication by using psychophysiological indicators. Such approach will allow us to reveal the general patterns of the distress-induced reactions to tragic historical events.

The following research questions (RQs) have been identified:

RQ 1: How do the watching historical documentaries containing real violence impact the EEG modulations of individuals?

RQ 2: Can ERPs serve as objective criteria of moral dilemma behavior?

We will conduct media effect studies on historicizing TV formats and investigate the following characteristics: impact of the historical media on physiological response, influence of the preliminary negative TVnews images on the following reactions. The excessive presentation of negative up-to-day images before the exposition of victim images in the context of the Holocaust could evoke defensive reactions, weariness or even aggression or desensitization. Therefore, there is a strong need to test empirically, which factors promote a humanity effect and which framework is required to account for successful comprehension of history. We addressed this need in this paper. 
The purpose of this study was to study the effect of negative emotional context created by the exposure of relevant images from TV news on the perception of target visual stimuli - victim images from the Holocaust documentary.

\section{Methods of research.}

\section{Participants}

38 healthy right-handed volunteers ( 21 women and $17 \mathrm{men}$ ), first-third year students of the Taras Shevchenko National University of Kyiv, Educational and Scientific Centre "Institute of Biology and Medicine" and Faculty of Psychology aged 18 to 27 years $\left(M_{\text {age }}=18.5, S D=2.06\right.$ years $)$ participated in this study. The participants were eligible to enroll in the study if they had normal or corrected-to-normal visual acuity, normal color vision, had no clinical manifestations of mental or cognitive impairment, verbal or non-verbal learning disabilities. Exclusion criteria were: the use of psychoactive medication, drug or alcohol addiction and psychiatric or neurological complaints.

The study was approved by the Bioethics Commission of Educational and Scientific Centre "Institute of Biology and Medicine", Taras Shevchenko National University of Kyiv and written informed consent was obtained from each subject in accordance with the World Medical Association (WMA) declaration of Helsinki - ethical principles for the medical research involving human subjects (Helsinki, Finland, June 1964).

\section{EEG recordings}

The EEGs were recorded monopolarly using EEG 23 Ch system Neurocom (Ukraine, XAI-MEDICA). The electrodes (silver/silver chloride) were placed on the scalp at symmetrical anterior frontal (Fp1, Fp2), frontal (F3, F4, Fz, F7, F8), central (C3, C4, Cz) parietal (P3, P4, Pz), occipital (O1, O2) and temporal (T3, T4, T5, T6) recording sites according to the international 10-20 scheme. All electrodes were referenced to the interconnected ear reference electrodes. The interelectrode impedance levels were below $5 \mathrm{k} \Omega$. The sample rate of all channels was 500 $\mathrm{Hz}$. A high-frequency filter with a $30 \mathrm{~Hz}$ cut-off frequency and a power network filter $(50 \mathrm{~Hz})$ were used; the time constant of the amplification tract was $0.3 \mathrm{sec}$.

\section{Stimuli}

For the current experiment we used the 3 sets of the images ( 80 frames in each set). Selection of the stimuli encompassed several steps in accordance with the principles, which we described earlier (13). First, we selected a pool of images from the neutral and negative TV news stories previously shown on TV and from the Holocaust documentary "Night and Fog" (1955, France). Then 4 coders ( 2 women and 2 men, aged 18 to 19 years $\left(M_{\text {age }}=18\right.$, $S D=0.5$ years), first grade students of the Taras Shevchenko National University of Kyiv, Educational and Scientific Centre "Institute of Biology and Medicine", assessed emotional valence of every images in the pool using a 10-grade "negative - positive" Likert-type scale (-5 - very negative, 0 - neutral, +5 - very positive). All coders' scores were averaged, classified in ascending order, and put within one of groups by negativity-positivity. We divided the whole scale span into tree parts: (1) negative images (valence values vary from -5 to -1 ), (2) neutral images (valence values vary from -1 to +1 ), (3) positive images (valence values from +1 to +5 ). As a result, we selected 80 negative TV news images, 160 neutral TV news images (randomly divided in half for two parts of the experiment) and 80 negative images from the Holocaust documentary.

\section{General Procedure of Trial}

The participant was seated in a comfortable armchair in a dimly lit recording booth in front of the standard 17" LCD monitor with a distance of $80 \mathrm{~cm}$ away from the computer screen. During the experiment, subjects were asked to look straight ahead and avoid extraneous movements. We recorded EEG using the following protocol. After adaptation to the study condition ( $2 \mathrm{~min}$ ) EEG was registered during the two periods of resting-state EEG, one 
with eyes closed $(3 \mathrm{~min})$, the other with eyes open $(1 \mathrm{~min})$ and during the presentation of images (total time of series - 6-7 $\mathrm{min})$.

The central processing of visual information depends on the emotional context. In two experiments we have studied effects of negative emotional context created by the exposure of relevant images from TV news on the perception of target visual stimuli - victim images from the Holocaust documentary. For this purpose we have divided the sample (38 healthy volunteers) into two groups. The first group (23 volunteers) was demonstrated a video set comprised of 80 negative images, selected from the Holocaust documentary "Night and Fog" (1955, France), and 80 neutral images. The second group (15 volunteers) was presented emotional frames taken from TV news plots (150 images) in order to investigate the pre-stimulus modulations of perception and processing of the emotional frames of historical documentaries.

We used in our study the simple discrimination task in "oddball" paradigm to investigate modulations of ERP components during the presentation and processing the emotional frames of TV news plots and historical documentary. Presentation time - $500 \mathrm{~ms}$, interval between stimuli $-2.4 \mathrm{sec}+/-30 \%$. The computer selected the image with a probability 50 to $50 \%(1: 1)$. Time analysis of stimuli - $150 \mathrm{~ms}$ before the presentation of images and $1000 \mathrm{~ms}$ after the beginning of the image presentation. During the exposure, event-related potentials (ERPs) were recorded. We analysed average signal amplitude of ERPs in the time intervals 40-80, 80-120, 120-220, 220-300, 300-400 and 400-700 ms after the onset of the exposure.

The processing of the data was carried out using the software package Neurocom (Ukraine, XAIMEDICA).

\section{Results of research.}

At the end of the experiment the participants assessed each set of images on the scales of "relaxing activating" and "unpleasant - pleasant". We noted that the emotional frames taken from negative TV news plots are relatively weak emotional stimuli compared to the emotional frames of historical documentary. The subjects exposed to the pre-stimulus evaluated the Holocaust pictures as more unpleasant and more activating than those without it (Table 1).

Table 1. Mean ratings of the emotionally neutral TV-news plots $(M \pm S D)$. Scales: -5 to +5

\begin{tabular}{|c|c|c|c|c|c|}
\hline Characteristics & $\begin{array}{c}\text { Frames taken } \\
\text { from negative } \\
\text { TV news plots }\end{array}$ & \multicolumn{2}{|c|}{$\begin{array}{c}\text { Frames taken from neutral TV } \\
\text { news plots }\end{array}$} & \multicolumn{2}{|c|}{$\begin{array}{c}\text { Images, selected from the } \\
\text { Holocaust documentary }\end{array}$} \\
\cline { 3 - 6 } & & $\begin{array}{c}\text { Without } \\
\text { negative } \\
\text { prestimuli factor }\end{array}$ & $\begin{array}{c}\text { With negative } \\
\text { prestimuli factor }\end{array}$ & $\begin{array}{c}\text { Without } \\
\text { negative } \\
\text { prestimuli } \\
\text { factor }\end{array}$ & $\begin{array}{c}\text { With negative } \\
\text { prestimuli } \\
\text { factor }\end{array}$ \\
\hline Pleasantness & $-1.87 \pm 1.48$ & $0.13 \pm 0.86$ & $-0.53 \pm 2.32$ & $-4.2 \pm 1.21$ & $-5.00 \pm 1.20$ \\
\hline $\begin{array}{c}\text { Arousal } \\
\text { potenial }\end{array}$ & $1.17 \pm 1.75$ & $0.27 \pm 0.78$ & $0.53 \pm 2.44$ & $2.53 \pm 1.6$ & $3.00 \pm 1.60$ \\
\hline
\end{tabular}

We have analyzed the evoked potentials recorded in the cortical areas that participate in the analysis of the visual stimulus, namely, the occipital lobe (visual cortex responsible for processing visual information), parietal areas (secondary and tertiary cortical associative fields of the visual analyzer), frontal cortex (responsible for the extraction of meaning, the content of the image, its evaluation, the formation of mental images and their actualization), temporal zones (cortical representation of the limbic system, which is associated with the processes of memory formation, perception and reproduction of emotions). 
We demonstrated that the ERPs recorded during the presentation of the negative (historical) and neutral images had various amplitudes of oscillations. The ERP amplitude of N170 (130-200 ms after stimulus presentation) and P300 (280-350 Mc) in frontal zones was bigger in response to the neutral pictures than to the negative historical ones. This contradicts the fact that the N170 reflects the neural processing of faces (1). The P300 component reflects conscious cognitive processes such as attention, decision-making and comparison of information (25), the activation of the memory trace after the detection of the target stimulus (34). We can assume that this indicates that the "abstract stimuli" are more familiar to the participants, but it takes greater effort to recognize them.

The historical images attracted more attention and were more emotional (occipital P300). This required more integration efforts to process the depicted information and to establish its semantic content. The images surprised the subjects (frontal N400) and activated their mental-psychological processes at the highest level of integration (process of analysis, retrieval of information from memory, semantic processes, namely search for the meaning of the pictures (late components (400-600 Mc) in the frontal and occipital zones).

We demonstrated that the ERPs recorded during the presentation of the negative (historical) images had various amplitudes of oscillations due to the preliminary affective impact of TV news frames.

The ERP amplitude of N200 (180-250 ms) and P300 in frontal zones was bigger for the group without the preliminary exposure to the emotional TV news frames due to the reduced sensitivity to the content of images. It is worthwhile to note in this case the decrease in the amplitude of N200 that arises during the perception and the arbitrary cognitive processing of stimuli (faces) (16). P300 allows for tracking down the timing of subjective evaluation and what exactly affects it. The direct relationship between latency P300 and the reaction time of the subject in the experiments indicates that P300 is part of the cognitive procedure that supports the formation of an internal model of the environment in which the stimulus is evaluated (hypothesis of context update) (24). In our case, the historical images demanded less attention and less emotional efforts for emotional evaluation of visual information, analysis, retrieval of information from memory and semantic processes, namely search for the meaning of the pictures (occipital P300 and LPP). Short-term media effects include alterations of sensitivity toward the emotional content of visual information.

Received data help clarify possible mechanisms involved in the perception of negative information. Our data are consistent with Moser \& colleagues (23) that shown emotion modulation of ERPs namely enhancement of the emotional responses to unpleasant stimuli. (23). We detected the negative stimulus-specific enhancement in amplitude of N400 ((250-500 ms)) and LPP in occipital zones in the response on the victim images from the Holocaust documentary. It should be noted that modulation refers to emotional (negative) stimuli. Bocanegra \& Zeelenberg, (2) indicated that emotional stimuli trigger a general enhancement in the efficiency of visual processing. Although emotional stimuli can weaken the perception of the subsequent neutral stimulus if two stimuli are present without a significant temporal difference due to the temporal competition for attention (2). Thus, the capture of attention by emotional stimuli can be accompanied by global inhibition of other representations in the cortex, and LPP can reflect this global inhibition.

The increase in the amplitude of component N400 (250-500 ms) under these conditions indicates analysis of meaningfulness of the visual stimuli (4). Emotional stimuli strongly modulate LPP (Late Positive Potential, 400$600 \mathrm{~ms}$ ) (28). The increase LPP in the amplitude in the frontal and occipital regions for images from the Holocaust documentary in a negative emotional context indicates the activation of the processing of incoming information in the top-down scenario, since the amplitude and latency of the LPP component increases with top-down processing of incoming information (22). It should be noted that top-down processing of sensory information is characterized by directed attention, caused by the perceptual anchoring of a new stimulus with a pre-formed hypothesis and 
expectations. LPP is associated with increased effectiveness of processing, building mental images, and recalling existing patterns for similar stimuli and paradigms (27).

\section{Conclusions}

Emotional visual stimuli are a complex substrate that requires the activation of numerous brain processes during processing incoming information. Negativity of stimuli (images from TV news) creates an emotional context for target visual stimuli - victim images from the Holocaust documentary and has an active impact on the perception processes that is expressed in modulating the level of attention and the processes of forming memory traces. Comparative analysis of ERP components found a significant influence of the context created by negative emotional stimuli, namely: the negative context promotes the processing incoming sensory information in the top-down scenario, which includes the activation of the mechanisms of memorization, selective attention.

\section{Competing interests}

The authors declare that they have no competing interests.

\section{References}

1. Blau, V. C., Maurer, U., Tottenham, N., \& McCandliss, B. D. (2007). The face-specific N170 component is modulated by emotional facial expression. Behavioral and brain functions, 3(1), 1. doi: https://doi.org/10.1186/1744-9081-3-7

2. Bocanegra, B. R., \& Zeelenberg, R. (2009). Dissociating emotion-induced blindness and hypervision. Emotion, 9(6), 865-873. doi: 10.1037/a0017749

3. Cruz, C., \& Bushman, B. J. (2014). Understanding media violence and its effects. Advertising and violence: Concepts and perspectives, 11-22.

4. Federmeier, K. D., \& Kutas, M. (2002). Picture the difference: Electrophysiological investigations of picture processing in the two cerebral hemispheres. Neuropsychologia, 40(7), 730-747. doi: https://doi.org/10.1016/S0028-3932(01)00193-2

5. Gavriely-Nuri, D., \& Lachover, E. (2012). Reframing the past as a cosmopolitan memory: Obituaries in the Israeli daily Haaretz. Communication Theory, 22(1), 48-65. DOI: 10.1111/j.1468-2885.2011.01397.x

6. Geipel, J., Hadjichristidis, C., \& Surian, L. (2015). How foreign language shapes moral judgment. Journal of Experimental Social Psychology, 59, 8-17. DOI: https://doi.org/10.1016/j.jesp.2015.02.001

7. Giles, D. (2003). Media psychology. Routledge.

8. Grimm, J. (2015). Value orientation and national identity in Russia: A media effect study on the Holocaust documentary "Night and Fog". Central European Journal of Communication, 8(1 (14)), 37-62.

9. Grimm, J., Rizun, V., Enzminger, A., Havrylets, Y., Tukaiev, S., Khylko, M., \& Nosova, B. (2016). Memorial Culture in Ukraine in the context of media perception of historical problems (based on documentaries about the Holocaust and Holodomor).Current Issues of Mass Communication, 20, 8-22. doi: https://doi.org/10.17721/2312-5160.2016.20.8-22 [Article in Ukrainian]

10. Grossman, L. C. D., \& DeGaetano, G. (2009). Stop teaching our kids to kill: A call to action against TV, movie \& video game violence. Harmony. 208 p.

11.Harrison, B. J., Pujol, J., Soriano-Mas, C., Hernández-Ribas, R., López-Solà, M., Ortiz, H., ... \& Segalàs, C. (2012). Neural correlates of moral sensitivity in obsessive-compulsive disorder. Archives of general psychiatry, 69(7), 741-749. DOI: 10.1001/archgenpsychiatry.2011.2165

12. Havrylets, Y. D., Tukaiev, S. V., Rizun, V. V., \& Makarchuk, M. Y. (2013). Comparative Analysis of the Effects of Negative and Neutral TV News Stories.Procedia-Social and Behavioral Sciences, 82, 421-425. doi: 10.1016/j.sbspro.2013.06.286 
13. Havrylets, Y., Rizun, V., Tukaiev, S., \& Khylko, M. (2016). Objectification of Subjectivity: International Experience in Selection of TV Stimuli in Mass Media Effect Research.Current Issues of Mass Communication, 19, 8-20 https://doi.org/10.17721/2312-5160.2016.19.8-20

14. Huesmann, L. R. (2007). The impact of electronic media violence: Scientific theory and research. Journal of Adolescent Health, 41(6), S6-S13. http://dx.doi.org/10.1016/j.jadohealth.2007.09.005

15. Kligler-Vilenchik, N., Tsfati, Y., \& Meyers, O. (2014). Setting the collective memory agenda: Examining mainstream media influence on individuals' perceptions of the past. Memory Studies, 7(4), 484-499. doi: $10.1177 / 1750698014523443$

16. Lange, J. J., Wijers, A. A., Mulder, L. J., \& Mulder, G. (1998). Color selection and location selection in ERPs: differences, similarities andneural specificity'. Biological psychology, 48(2), 153-182. doi: https://doi.org/10.1016/S0301-0511(98)00011-8

17. Leggewie, C., \& Lang, A. (2011). Der Kampf um die europäische Erinnerung: ein Schlachtfeld wird besichtigt. München: C.H. Beck.

18. Levy, D., \& Sznaider, N. (2002). Memory Unbound The Holocaust and the Formation of Cosmopolitan Memory. European Journal of Social Theory, 5(1), 87-106. doi: 10.1177/1368431002005001002

19. Levy, D., \& Sznaider, N. (2006). The Holocaust and memory in the global age (Vol. 61). Temple University Press.

20. Liu, J. H., Goldstein-Hawes, R., Hilton, D., Huang, L. L., Gastardo-Conaco, C., Dresler-Hawke, E., ... \& Kashima, Y. (2005). Social representations of events and people in world history across 12 cultures. Journal of cross-cultural psychology, 36(2), 171-191. doi: 10.1177/0022022104272900

21. Liu, J. H., \& Hilton, D. J. (2005). How the past weighs on the present: Social representations of history and their role in identity politics. British Journal of Social Psychology, 44(4), 537-556. DOI: $10.1348 / 014466605 \times 27162$

22. Moradi, A., Mehrinejad, S. A., Ghadiri, M., \& Rezaei, F. (2017). Event-related potentials of bottom-up and top-down processing of emotional faces. Basic and clinical neuroscience, 8(1), 27-36. doi: https://doi.org/10.15412/J.BCN.03080104

23. Moser, J. S., Hajcak, G., Bukay, E., \& Simons, R. F. (2006). Intentional modulation of emotional responding to unpleasant pictures: an ERP study. Psychophysiology, 43(3), 292-296. doi: https://doi.org/10.1111/j.1469-8986.2006.00402.x

24. Oliver-Rodriguez, J. C., Guan, Z., \& Johnston, V. S. (1999). Gender differences in https://doi.org/10.1111/1469-8986.3620176 late positive components evoked by human faces. Psychophysiology, 36(2), 176-185. doi: https://doi.org/10.1111/1469-8986.3620176

25. Polich, J. (2007). Updating P300: an integrative theory of P3a and P3b. Clinical neurophysiology, 118(10), 2128-2148. doi: https://doi.org/10.1016/j.clinph.2007.04.019

26. Roediger, H. L., \& Abel, M. (2015). Collective memory: a new arena of cognitive study. Trends in cognitive sciences, 19(7), 359-361. DOI: http://dx.doi.org/10.1016/j.tics.2015.04.003

27. Ruchkin, D. S., Johnson Jr, R., Mahaffey, D., \& Sutton, S. (1988). Toward a functional categorization of slow waves. Psychophysiology, 25(3), 339-353. doi: https://doi.org/10.1111/j.1469-8986.1988.tb01253.x

28. Sabatinelli, D., Lang, P. J., Keil, A., \& Bradley, M. M. (2006). Emotional perception: correlation of functional MRI and event-related potentials. Cerebral cortex, 17(5), 1085-1091. doi: https://doi.org/10.1093/cercor/bh1017 
29. Sagi-Schwartz, A. (2015). Does Extreme Trauma Transfer? The Case of Three Generations of the Holocaust. In Traumatic Stress and Long-Term Recovery: Coping with Disasters and Other Negative Life Events (pp. 133-148). Springer International Publishing.

30. Silbermann, A., \& Stoffers, M. (2000). Auschwitz. Nie davon gehört?: Erinnern und Vergessen in Deutschland. Rowohlt Berlin.

31. Üngör, U.U . (2014) Lost in commemoration: the Armenian genocide in memory and identity.Patterns Prejudice,48, 147-166. DOI: http://dx.doi.org/10.1080/0031322X.2014.902210

32. Zuckermann, M. (1999). Zweierlei Holocaust: der Holocaust in den politischen Kulturen Israels und Deutschlands. Wallstein Verlag.

33. Zuckermann, M. (2004). Zweierlei Holocaust. Der Holocaust in den politischen Kulturen Israels und Deutschlands, 3. Aufl. (zuerst 1998). Göttingen: Wallstein Verlag.

34. Verleger, R. (1988). From epistemology to P3-ology. Behavioral and brain sciences, 11(3), 399-408. doi: https://doi.org/10.1017/S0140525X00058234 